\title{
Early Institutional Head and Neck Oncologic and Microvascular Surgery Practice Patterns Across the United States During the SARS-CoV-2 (COVID19) Pandemic
}

\author{
Rusha J. Patel et al. ${ }^{1}$ \\ ${ }^{1}$ Affiliation not available
}

April 28, 2020

\begin{abstract}
Background: The SARS-CoV-2 (COVID-19) pandemic has caused rapid changes in head and neck cancer (HNC) care. 'Realtime' methods to monitor practice patterns can optimize provider safety and patient care.Methods: Head and neck surgeons from 14 institutions in the United States regularly contributed their practice patterns to a shared spreadsheet. Data from March $27^{\text {th }}, 2020$ to April $5^{\text {th }}$, 2020 was analyzed.Results: All institutions had significantly restricted HNC clinic evaluations. 2 institutions stopped free flap surgery with the remaining scheduling surgery by committee review. Factors contributing to reduced clinical volume included lack of personal protective equipment (PPE) (35\%) and lack of rapid COVID-19 testing (86\%).Conclusions: The COVID-19 pandemic has caused a reduction in HNC care. Rapid COVID-19 testing and correlation with infectious potential remain paramount to resuming the care of head and neck cancer patients. Cloud-based platforms to share practice patterns will be essential as the pandemic evolves.
\end{abstract}

\section{INTRODUCTION}

The COVID-19 pandemic, caused by SARS-CoV-2, has been implicated in over 360,000 cases in the United States alone and has been found to affect as many as $28 \%$ of HCW worldwide ${ }^{1,2}$. Physicians across specialties have had to make rapid decisions about personal protective equipment (PPE), patient care triaging, and navigating national and international guidelines that continue to evolve. These decisions are not uniform and vary within the context of individual location's infection rate and available resources.

This is of special concern to otolaryngologists, who are among the highest risk specialties for COVID-19 exposure from nasal and mucosal procedures and examinations ${ }^{3,4}$. The highest concentration of viral particles is found in the nasopharynx and routine procedures performed by otolaryngologists can easily aerosolize viral particles and allow for airborne trasmission ${ }^{5,6}$. In these early days of the pandemic, recommendations to keep both patients and providers safe were made based on data from Wuhan, China, northern Italy, and extrapolations from the SARS-CoV-1 epidemic. Givi et al distilled these recommendations into specifics regarding protective equipment (PPE) and practice considerations for head and neck surgeons during the COVID-19 pandemic ${ }^{7}$. The implementation of these recommendations has been variable across institutions, likely based upon regional COVID19 case load and resource availability.

As a subspecialty, head and neck cancer surgeons have had to balance infection risk with patient care. Surgery remains a mainstay for head and neck cancer treatment, but poses a high risk of viral exposure. Surgical treatment delays have been shown to significantly increase the risk of recurrence and reduce overall survival $^{8,9}$. Thus, head and neck surgeons are tasked with triaging patient care and balancing their decisions with the safety of themselves, their team, and support staff. Cancer patients have significantly higher rates of 
morbidity and mortality if infected with the novel coronoavirus, but a cohesive approach to testing patients and weighing the risks of patient exposure with resource utilization and patient survival is lacking ${ }^{10}$.

In the absence of readily available peer-reviewed information, North American head and neck surgeons discussed the complicated issue of protecting the providers, operating room staff, clinic staff and patients via email chains, social media messaging platforms, message boards, and text groups. Sensing this need for 'real-time' information, institution-specific data on head and neck surgery practice patterns during the COVID19 pandemic was collected and distilled into an accessible spreadsheet. Here is presented the data from 14 different institutions, focusing specifically on early practice patterns related to head and neck surgery and patient care.

\section{METHODS}

A shared spreadsheet with no patient-specific data included was created with Google Docs. Contributors, all otolaryngology/head and neck surgeons practicing in the United States, were solicited via email and text. All contributors were given the option of remaining anonymous. Data collected included current COVID-19 burden in the state, PPE practices, perioperative COVID-19 testing, cancer case scheduling concerns, and utilization of residency cadres. Information gathered is updated regularly and available on the American Head and Neck Society (AHNS) Bulletin Board at https://www.ahns.info/covid-19-info/. This study is based on data collected from March $27^{\text {th }}, 2020$ to April $5^{\text {th }}, 2020$. Data were converted into discrete variables and presented numerically and as percentages.

\section{RESULTS}

Fourteen institutions from multiple tertiary care sites across the country contributed to data analysis. The highest COVID-19 burdens were reported from three programs in California, with over 12,267 cases in the state at the time of analysis. The lowest was from West Virginia with 282 cases reported. Practice pattern results are summarized in Figure 1.

In-house COVID-19 testing was available at 12 of 14 institutions, but was limited at one site. Ten institutions (71\%) were performing pre-operative COVID-19 testing, with rapid testing available at two institutions.

High-risk procedures for COVID-19 transmission were defined as mucosal, endoscopic, or aerodigestive surgery (including tracheostomy) by all institutions. Six institutions included mastoid surgery as high-risk, and two additionally defined salivary surgery as a high-risk procedure. 10 institutions were using N95 masks with a face shield in addition to standard operating room PPE for high-risk procedures in COVID-19 tested negative patients. One of these institutions additionally used a powered air-purifying respirator (PAPR) for prolonged high-risk cases such as free tissue transfer. The remaining four institutions used standard surgical PPE alone for COVID-19 tested negative patents. For COVID-19 positive patients, 3 institutions used a PAPR. The remaining 11 institutions used either a PAPR or an N95 mask (Figure 2).

Twelve institutions (83\%) were continuing free flap surgery. One institution was continuing on a limited basis, and the other two had temporarily stopped free flap surgery. Most institutions had implemented a review process for cancer surgery scheduling (Figure 3). Ten institutions were using a case review by another individual or a multidisciplinary committee. Two institutions were reviewing cases via regular tumor board meetings. The remaining two institutions were asking surgeons to limit cases to those he or she deemed essential. All institutions reported prioritizing cancer cases that could not be delayed 6-12 weeks. Transoral robotic surgery (TORS) was temporarily deferred at four institutions. Most institutions reported using an N95 mask for microvascular anastomoses. Five institutions reported that a lack of appropriate PPE had interfered in their ability to continue surgical cases. Thirteen of 14 institutions reported intentionally limiting resident participation in high-risk cases.

All institutions had limited clinical visits to those that were urgent or selected by the provider. All but one institution were using telemedicine for clinic appointments; one institution had the platform pending. No institutions were offering antibody testing for providers at the time of analysis. At the time of analysis, 
no providers had been reassigned to alternate health care roles. Four institutions reported providers being queried about reassignment should the need arise.

\section{DISCUSSION:}

The COVID-19 pandemic has shifted the structure of health care rapidly and continues to do so on a daily basis. In the absence of precedent, physicians have turned to each other to determine best practices and to prepare for the long-term effects of the COVID-19 pandemic.

Utilizing multiple platforms, head and neck surgeons have been able to rapidly disseminate information regarding evolving important updates to practice patterns that allow for optimal patient care in times of crisis. Not all platforms are equal: text and email can provide one-time narrative information; social media, while useful for the rapid exchange of information, can propagate false or misleading information which can be easily perpetuated and place providers at risk for retaliation from their institution. We created an anonymous, secure, and reliable method to allow for real-time information regarding head and neck cancer care. This system is modular and has provided valuable data about current practice patterns and changes in these patterns as institutions and guidelines adjust.

The majority of reporting institutions had implemented in-house COVID-19 testing abilities and followed similar PPE practices, including the use of an N95 mask for high-risk procedures. Several institutions recommended a personal air purifying device (PAPR) for patients with unknown or positive COVID-19 status. PAPRs are reusable, but not widely available. The PPE needs of major head and neck oncologic surgery depend both on rapid and accurate COVID-19 testing, and a determination of the true infectious risk of head and neck procedures. 35\% of institutions reported lack of PPE prevented their surgical care. Until testing results and infectious risk are shown to correlate, head and neck surgery may continue to be prohibitively resource-heavy during the COVID-19 pandemic. Rapid pre-operative testing, such as that reported by two institutions, has some promise in mitigating PPE needs but this remains to be assessed.

Our early results additionally show a significant decrease in the volume of clinical care provided to head and neck cancer patients. Patients seen in clinic have an 'unknown' COVID-19 status, placing staff and providers at high risk from a standard head and neck exam. As such, the majority of institutions limited in-person evaluations to urgent visits. While most institutions had implemented telemedicine, this format precludes a full mucosal site exam and therefore has intrinsic limitations in the evaluation and follow-up of head and neck cancer patients. As the duration of limited clinical evaluation continues, consideration will have to be given as to how safely evaluate new head and neck cancer patients and effectively provide surveillance to patients who have recently completed treatment. This could include a reliable method of pre-clinical symptom screening, such as that currently being performed on providers at three reporting institutions.

Adding to the gap in head and neck cancer care, we found that several institutions in areas of higher COVID19 burden had severely limited or temporarily halted head and neck surgery requiring free tissue transfer. As infection rates in other regions rise there is a potential for more institutions to be unable to support these procedures. The majority of institutions that were proceeding with free flap surgery were working with limited intraoperative assistance and were scheduling via a review process that could potentially delay surgery pending more urgent operating room needs. Major head and neck oncologic surgery is resourceintense from the perspectives of PPE use, critical care support, and blood blank supplies. However, these surgeries are not only important for improved function, they are often the best or only option for oncologic control in patients suffering from head and neck cancer. Triaging cancer cases in the setting of a pandemic is an unfortunate reality. Specialty societies such as the American Head and Neck Society (AHNS), Society of Endocrine Surgeons, the American College of Surgeons, and the Society of Surgical Oncology have provided useful guidance to support these difficult decisions being made by surgeons across the country ${ }^{11,12,13}$. As surgical resources continue to be limited, serious multidisciplinary consideration will need to be given to alternate methods of head and neck cancer care. The decision to suspend these procedures during this time of crisis places an immeasurable ethical burden on surgeons who have dedicated their lives to the care of head and neck cancer patients, and incredible potential for morbidity in patients who cannot receive their 
anticipated care.

Peer-reviewed data is not available to guide head and neck surgeons through this pandemic when it is most needed. Real-time data sharing has allowed head and neck surgeons to share and improve local practices in order to mitigate harm and prevent the spread of infection. Our data show that head and neck cancer care has been restricted, and will likely continue to be restricted as COVID-19 incidences rise across the country. The long-term effects on survival remain unknown, and the greater need of preserving health care resources to the population during the COVID-19 pandemic remains a priority while infection rates continue to rise. Current focus should be on rapid and accurate perioperative COVID-19 testing to mitigate PPE needs, allowing for similar testing and/or screening practices to resume clinic evaluations, and focusing on methods of least-harm during times of limited surgical care. We hope this platform will continue to evolve and provide valuable information to head and neck surgeons as the pandemic progresses.

\section{REFERENCES}

1. Gardner LD, Ensheng and Johns Hopkins Center for Systems Science and Engineering. Coronavirus COVID-19 Global Cases by the Center for Systems Science and Engineering (CSSE) at Johns Hopkins University (JHU). Available at: https://coronavirus.jhu.edu/map.html. 2020.

2. Jacob B Aguilar JSF, Lauren M. Westafer, Juan B. Gutierrez. Investigating the Impact of Asymptomatic Carriers on COVID-19 Transmission.medRxiv 2020; 20037994.

3. Dan Lu, Haiyang Wang, Rong Yu et a;. Integrated infection control stragety to minimize nosocomial infection of corona virus disease 2019 amount ENT healthcare workers. Journal of Hospital Infection. 2020 Feb 22 (pre-proof): https://www.ahns.info/wp-content/uploads/2020/03/Wuhan-ENTExperience.pdf

4. Patel, S et al. "Stanford Commentary for Nasal Procedures in COVID-19 era" https://www.ahns.info/wpcontent/uploads/2020/03/Stanford-Commentary-for-Nasal-procedures-in-COVID-19-era.pdf

5. Zou L, Ruan F, Huang Met al. SARS-CoV-2 Viral Load in Upper Respiratory Specimens of Infected Patients. N Engl J Med 2020; 382:1177-1179.

6. van Doremalen N BT, Morris DH, et al. Aerosol and Surface Stability of SARSCoV-2 as Compared with SARS-CoV-1. N Engl J Med 2020.

7. Givi B, Schiff B, Chinn S, et al. "Safety recommendations for evaluation and surgery of the head and neck during the COVID-19 pandemic JAMA Head and Neck [REF]

8. Jensen AR1, Nellemann HM, Overgaard J. Tumor progression in waiting time for radiotherapy in head and neck cancer. Radiother Oncol. 2007 Jul;84(1):5-10

9. Chen MM, Harris JP, Orosco RK et al. Association of Time between Surgery and Adjuvant Therapy with Survival in Oral Cavity Cancer. Otolaryngol Head Neck Surg. 2018 Jun;158(6):1051-1056.

10. Liang W, Guan W, Chen R et al. Cancer patients in SARS-CoV-2 infection: a nationwide analysis in China. The Lancet Oncology. 2020 March 1; 21(3): 335-337

11. Ueda M, Martins R, Hendrie PC et al. Managing Cancer Care During the COVID-19 Pandemic: Agility and Collaboration Toward a Common Goal. J Natl Compr Canc Netw. 2020 Mar 20:1-4

12. "COVID-19: Guidance for Triage of Non-Emergent Surgical Procedures" https://www.facs.org/covid19/clinical-guidance/triage

13. Randolph G, Stack B. "Endocrine Surgery during the Covid-19 pandemic" file://C:/Users/H67312/AppData/Local/Mi Surgery-during-the-Covid.pdf

\section{Full author list}

Rusha J. Patel, MD FACS ${ }^{1}$; Alexandra Kejner, MD FACS ${ }^{2}$; Caitlin McMullen, MD FACS ${ }^{3}$

1. West Virginia University, Morgantown, West Virginia

2. University of Kentucky Markey Cancer Center, Lexington, KY

3. Moffitt Cancer Center, Tampa, FL

Corresponding Author: Rusha J. Patel1 Medical Center Drive PO Box 9200 Morgantown, WV 26505 Rusha. 
patel@hsc.wvu.edu O: 304-293-3457

Acknowledgements: The authors would like to acknowledge the contributing members of each institution, both past and future, as well as the American Head and Neck Society for their support.

\section{Figures}

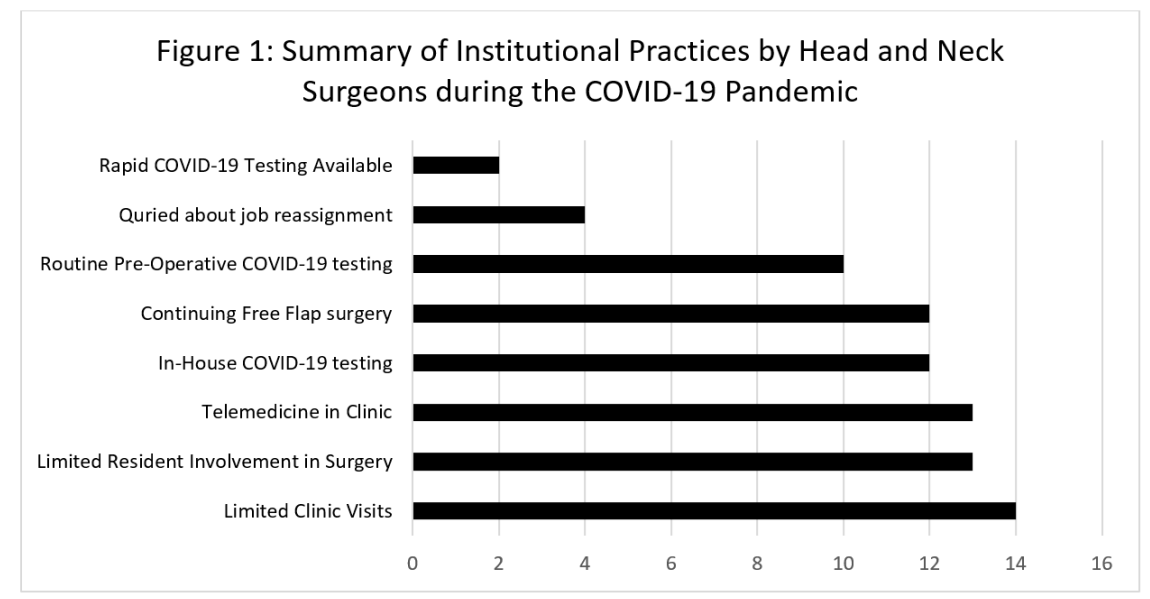

Figure 1: Summary of Institutional Practices by Head and Neck Surgeons during the COVID-19 Pandemic.

Figure 2: Institutional Personal Protective Equipment (PPE) for High-Risk Surgery

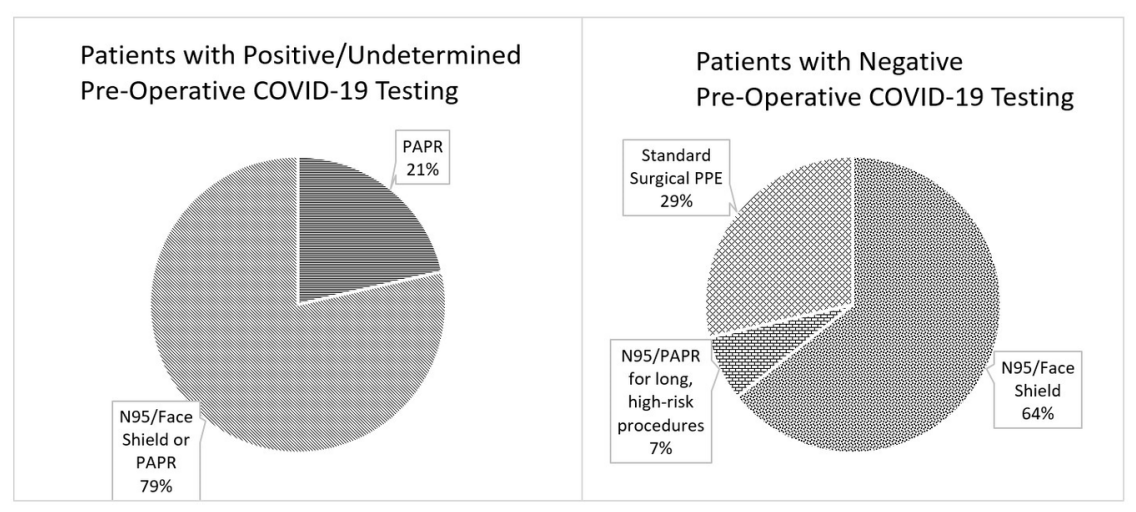

PAPR: powered air purifying respirator

Figure 2: Personal Protective Equipment (PPE) for High-Risk Surgical Procedures 


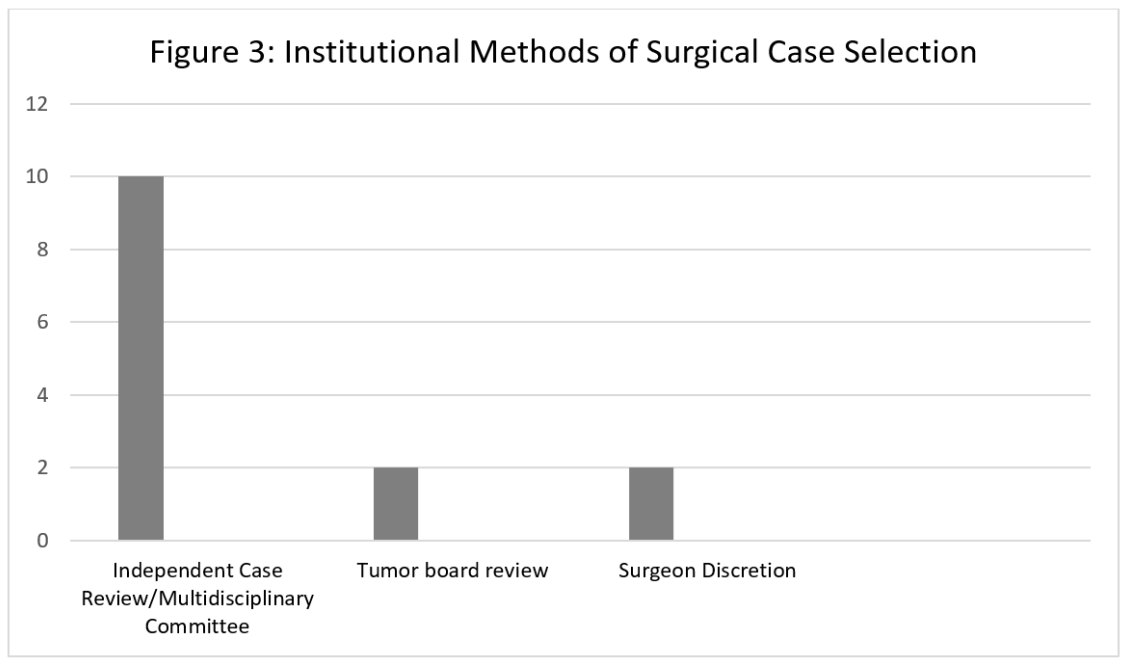

Figure 3: Institutional Methods of Surgical Case Selection 